\title{
La mise en circulation des objets scientifiques
}

Organisation pratique et changements de statut. Le cas des escargots patrimoniaux du Mercantour

The circulation of scientific objects : practical organization and changes in status. The case of the Mercantour's rare snails

Isabelle Mauz

\section{(2) OpenEdition}

Journals

Édition électronique

URL : https://journals.openedition.org/tc/6666

DOI : $10.4000 /$ tc. 6666

ISSN : 1952-420X

Éditeur

Éditions de l'EHESS

Édition imprimée

Date de publication : 15 décembre 2012

Pagination : 224-241

ISBN : 978-2-7351-1534-1

ISSN : 0248-6016

\section{Référence électronique}

Isabelle Mauz, «La mise en circulation des objets scientifiques », Techniques \& Culture [En ligne], 59 |

2012, mis en ligne le 15 décembre 2015, consulté le 29 septembre 2022. URL : http://

journals.openedition.org/tc/6666 ; DOI : https://doi.org/10.4000/tc.6666 


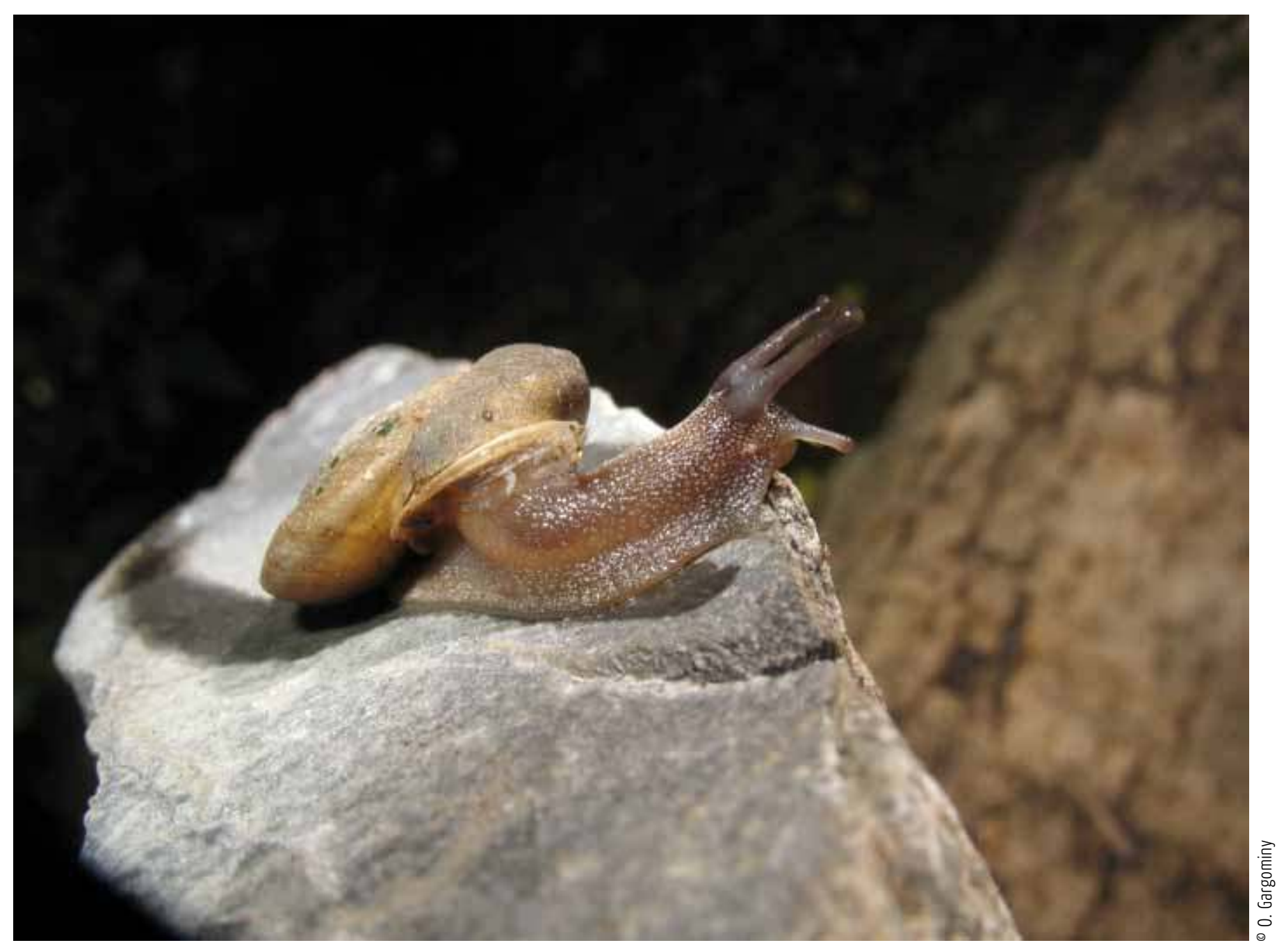




\section{LA MISE EN CIRCULATION DES OBJETS SCIENTIFIQUUES}

\section{Organisation pratique et changements de statut. Le cas des escargots patrimoniaux du Mercantour}

Les escargots dits patrimoniaux du Mercantour appartiennent à des espèces endémiques qui se caractérisent par une très faible mobilité. Ils vivent dans de minuscules biotopes (comme des anfractuosités de rochers) dont ils ne sortent pas et qu'ils paraissent habiter depuis des temps très reculés (Figure 1). Ayant intéressé des systématiciens du Muséum national d'histoire naturelle (MNHN) et des agents du parc national du Mercantour, ces escargots, ou du moins leurs coquilles, se sont mis à voyager. Collectées sur un coin de rocher de la vallée de la Roya, de la Tinée ou de la Vésubie, des coquilles ont été expédiées au siège du parc national, à Nice, et, pour certaines, au laboratoire de malacologie du Muséum, à Paris. Quelques-unes ont rejoint l'armoire à tiroirs du laboratoire, qui héberge la collection nationale de référence de mollusques terrestres. Elles y ont repris une existence extrêmement sédentaire (Figure 2). À cette circulation matérielle bien circonscrite dans l'espace et dans le temps s'est ajoutée une circulation virtuelle : les escargots endémiques du Mercantour côtoient des mollusques du bout du monde sur les écrans d'ordinateur des malacologues, leurs descriptions et leurs photographies transitent sur Internet. Cet article s'appuie sur l'exemple de ces escargots pour réfléchir à l'organisation et aux effets de la mise en circulation des objets scientifiques entre des mondes sociaux différents.

La malacologie est propice à l'exploration sociologique de cette mobilisation ${ }^{1}$. L'hétérogénéité du travail scientifique y est en effet souvent très poussée. Ainsi, les anthropologues ont mis en évidence l'implication simultanée de chercheurs du Muséum, d’amateurs de haut niveau, de techniciens, de plongeurs, de collectionneurs-marchands et d'une partie des habitants des régions prospectées dans la collecte des mollusques marins, lors des expéditions organisées par le MNHN et l'association Pro-Natura (voir Demmer, Dumoulin et Faugère dans ce numéro). 


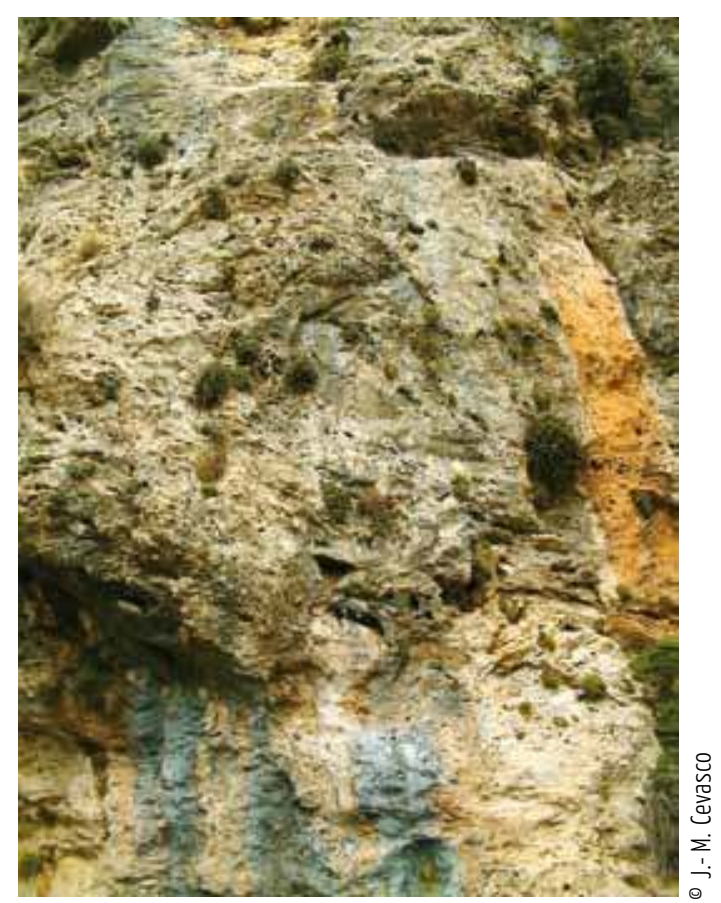

D’un microbiotope... (Fig. 1)
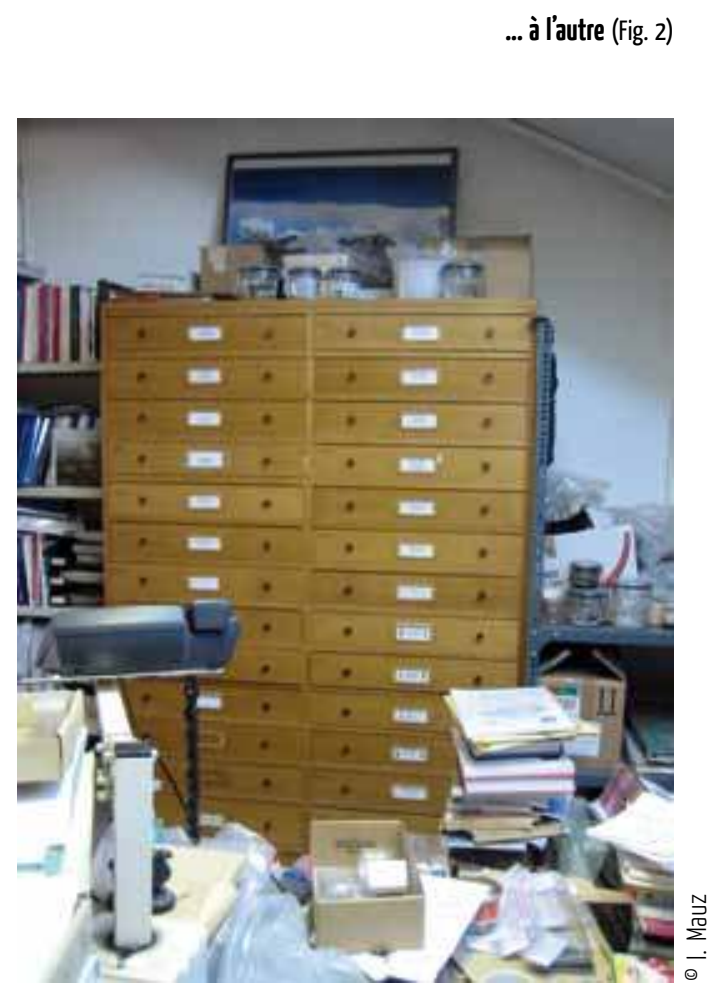

Cette hétérogénéité du travail scientifique se vérifie, à un moindre degré, dans le cas d'étude qui nous occupera ici : des systématiciens du MNHN et des agents du parc national ont étroitement collaboré pour effectuer un inventaire des escargots patrimoniaux du Mercantour. À condition de garder à l'esprit que la réalité est plus mêlée, on peut dire que les premiers appartiennent au monde de la recherche académique, les seconds au monde de la protection de la nature. Comment les coquilles ont-elles été amenées à circuler d'un monde professionnel à l'autre ? Et avec quels effets sur les coquilles et sur ceux qui les ont mises en mouvement?

La réflexion s'appuie sur une enquête menée de 2010 à 2012, qui a visé à retracer les itinéraires des coquilles d'escargots, du Mercantour au Muséum et à saisir les transformations qui les ont accompagnés. Suivant la démarche de l'ethnographie multi-située (Marcus 1995), j’ai reconstitué les trajets des coquilles d'escargot et des fiches qui leur sont associées, du terrain où elles ont été respectivement collectées et remplies jusqu'au laboratoire de malacologie du Muséum, en passant par le siège du Parc à Nice. J'ai conduit sept entretiens semi-directifs auprès de malacologues du Muséum et d'agents du parc national fortement impliqués dans l'inventaire des escargots patrimoniaux du Mercantour effectué au début des années 2000. Les entretiens ont été complétés par une consultation des documents disponibles (guide d'identification des escargots patrimoniaux du Mercantour, conventions entre MNHN et Parc, rapports d'étude et articles scientifiques des systématiciens, sites internet de malacologie) et par une observation des collections de coquilles constituées en différents lieux (Figure 3).

Le texte comporte quatre parties. Je commencerai par introduire les principales notions sociologiques utilisées : celle d'objet-frontière (Star et Griesemer 1989) et celle de vie sociale des choses (Appadurai 1986). Je présenterai ensuite la genèse et le déroulement de l'inventaire des escargots patrimoniaux du Mercantour. Je m'intéresserai alors à l'organisation de la mise en circulation des coquilles : je pointerai ses dimensions matérielle, institutionnelle et marchande et l'existence simultanée de règles de circulation et d'accidents de parcours. La dernière partie portera sur les changements de statut opérés par la mobilisation des objets scientifiques. On verra notamment que les transformations des personnes qui y participent constituent à la fois une motivation forte pour contribuer à l'effort d'inventaire naturaliste et une source potentielle de frictions.

\section{La vie sociale des objets-frontières}

Le processus d'accumulation des connaissances scientifiques repose sur une mobilisation massive d'objets, depuis la multitude de sites où ils sont collectés ou observés jusqu'à un nombre beaucoup plus 
réduit de lieux, où ces objets ou des renseignements à leur sujet sont rassemblés, étudiés, mis en chiffre et en équation, etc. (Latour 1989 : chapitre 6).

Cependant, les chercheurs ne sont pas capables, à eux seuls, de mettre ainsi les objets en mouvement pour les amener là où ils peuvent les mettre à plat et les analyser à loisir. L'entreprise scientifique est toujours marquée par une coopération entre des personnes qui appartiennent à des mondes sociaux différents (Star et Griesemer 1989). Certes, ces mondes sociaux ne sont pas entièrement disjoints ni étanches. Par exemple, on peut être, simultanément ou successivement, chercheur et naturaliste amateur, chercheur et gestionnaire, gestionnaire et naturaliste amateur. Comme je l'ai déjà suggéré, parler du monde de la recherche académique et du monde des naturalistes amateurs, ou du monde des gestionnaires de la nature est une simplification d'une réalité complexe. Il s'agit d’un modèle de la réalité, volontiers utilisé par les personnes pour décrire et commenter les interactions dans lesquelles elles sont engagées et que les sociologues trouvent commode de reprendre.

Les chercheurs en Science and Technology Studies ont cherché à comprendre comment des personnes appartenant à des mondes aux pratiques, aux valeurs et aux objectifs partiellement différents parviennent à collaborer. Star et Griesemer (id.) ont étudié comment des acteurs et des groupes d'acteurs ont coopéré pour constituer un musée d'histoire naturelle fortement tourné vers la recherche scientifique. Ils ont avancé l'idée selon laquelle cette coopération a été permise d'une part par l'adoption de méthodes standardisées (O'Connell 1993), d'autre part par des objets qui passent d'un monde social à l'autre. Ils ont donné de ces "objets-frontières" (boundary objects) une définition devenue classique ${ }^{2}$ : « objects which are both plastic enough to adapt to local needs and the constraints of the several parties employing them, yet robust enough to maintain a common identity across sites » (Star et Griesemer 1989 : 393).

Les deux explications proposées par Star et Griesemer valent dans le cas présent : la coopération entre les gestionnaires du Parc et les systématiciens du Muséum a reposé, en effet, sur une standardisation des méthodes de relevé et d'étiquetage des coquilles et sur la circulation, d'un monde à l'autre, des objets-frontières que sont les coquilles d'escargots et les fiches de relevé. Mais l'enquête menée suggère que la mise en circulation de ces objets a opéré une transformation de leur statut et de ceux qui les ont mis en mouvement. Il semble alors que la mise en circulation d'objets-frontières ne permette pas seulement la coopération entre des mondes sociaux différents mais contribue également à la reconfiguration de ces mondes et de leurs interrelations. Outre la question des effets de la mobilisation des objets scientifiques, se pose aussi la question de l'organisation pratique de cette mobilisation entre des mondes sociaux différents.

Les recherches sur « la vie sociale des choses » initiées par Appadurai me seront utiles pour explorer ces deux questions - comment s'opère, concrètement, la mobilisation des objets scientifiques et quels changements de statut produit-elle? Appadurai (1986) a défendu l'idée selon laquelle les objets ont une trajectoire biographique, à l'instar des 


\section{Cochlostoma simrothi (Caziot, 1908)}

Famille : Cochlostomatidae (Gastropoda)

Statut patrimonial : Sub-endémique (France - Italie)

Identification :

Escargots à opercule, les Cochlostomes sont des especes facilement reconnaissables par leur coquille conique, généralement finement striée, et leur péristome (ouverture) arrondi. Lidentification des espèces est plus délicate (voir figure ci-dessous pour les espèces présentes dans le secteur Roya).

\section{Habitat :}

Cette espèce vit collée sur les rochers calcaires, dans les vallées.

Décrite des Gorges de Saorge, dans les Alpes-Maritimes, cette espèce est endémique des parties moyenne et supérieure de la vallée de la Roya et de la partie supérieure du Val Nervia, dans la province d'Imperia (Italie).
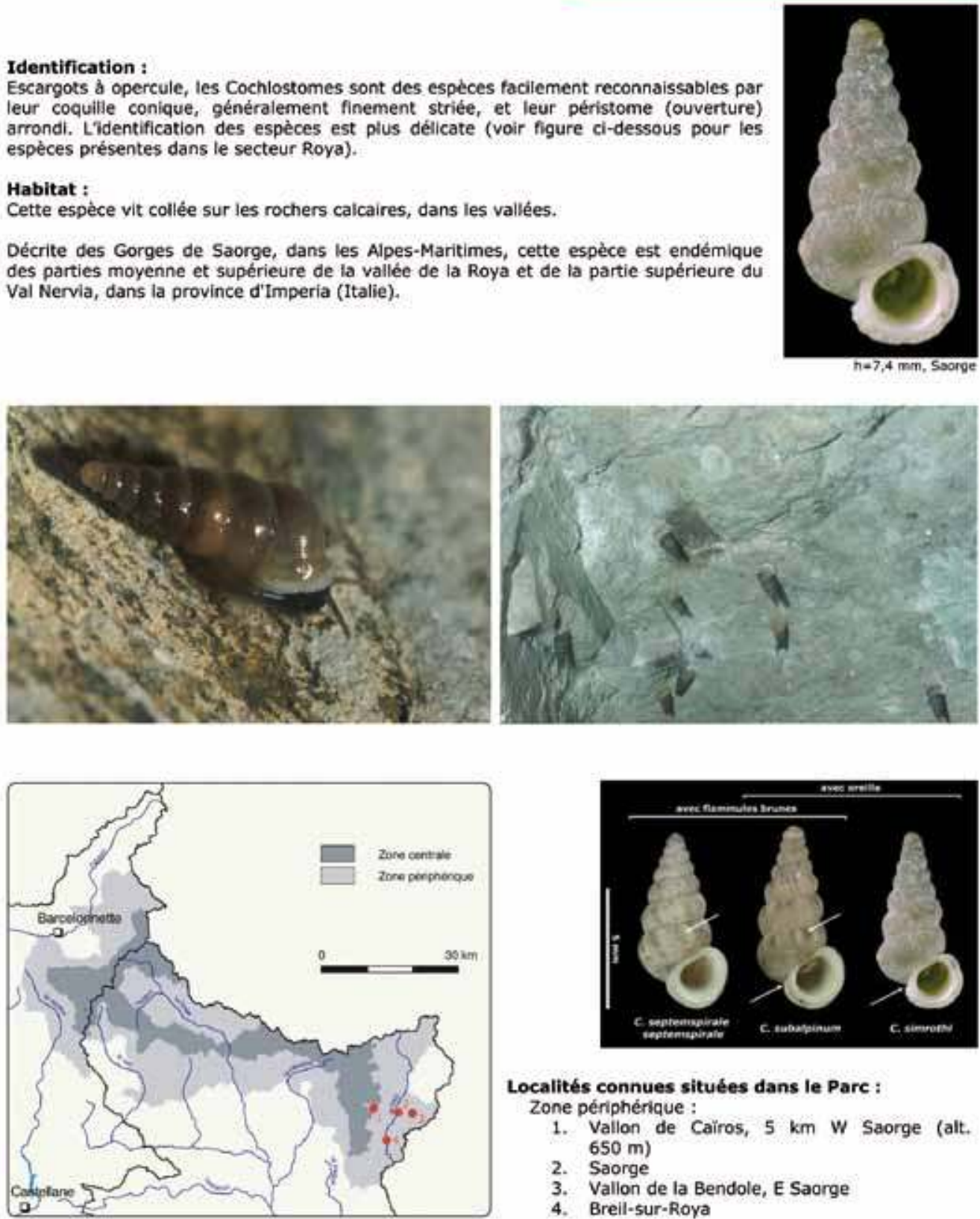

Localités connues situées dans le Parc:

Zone périphérique :

1. Vallon de Cairos, $5 \mathrm{~km} \mathrm{~W}$ Saorge (alt. $650 \mathrm{~m}$ )

2. Saorge

3. Vallon de la Bendole, E Saorge

4. Breil-sur-Roya 
êtres vivants. Sa réflexion a plus précisément porté sur la façon dont les objets peuvent devenir des marchandises à un moment donné de leur existence et cesser de l'être par la suite (Kopytoff 1986 : 64-91). Il s'agit alors moins de décider, de manière plus ou moins arbitraire, si un objet est, ou pas, une marchandise que d'étudier les processus de marchandisation et de dé-marchandisation. Ces processus font aussi partie de l'activité scientifique : il faut bien financer le travail des chercheurs et l'achat des équipements dont ils ont besoin. Des recherches menées et initiées par Appadurai, je retiendrai surtout que les objets changent de statut en passant d'un acteur et/ou d'un lieu à un autre. Leur trajectoire, telle qu'on peut l'observer en suivant les objets, se rapproche ou au contraire s'écarte plus ou moins d'une trajectoire considérée comme idéale. Les coquilles d'escargots patrimoniaux collectées dans le Mercantour et les fiches de relevé n’ont pas nécessairement suivi l'itinéraire tout tracé qui devait les mener d'un coin de rocher à la collection nationale de référence du Muséum : certaines ont connu ce que l'on peut appeler des accidents de parcours. Mais nous n'en sommes pas là ; il nous faut d'abord présenter la genèse et le déroulement de l'inventaire qui a mis ces animaux singulièrement lents et peu mobiles en mouvement.

\section{Genèse et déroulement de l'inventaire des escargots patrimoniaux du Mercantour (2000-2006)}

Les enquêtés ont désigné comme origine de l'inventaire un article de Philippe Bouchet, qui identifie les Alpes maritimes comme un des hauts-lieux de l'endémisme des mollusques terrestres en France métropolitaine (Bouchet 1990). À la suite de cet article, deux malacologues du Muséum ont entrepris un inventaire des mollusques d'intérêt patrimonial de la région Provence Alpes Côte d'Azur (Gargominy et Ripken 1999). Les membres du service Étude et Gestion des Milieux (EGM) du parc national du Mercantour ont repéré l'article de Philippe Bouchet et ont échangé avec l'un des auteurs de l'inventaire lors de la présentation de ce travail au Comité Scientifique Régional de Protection de la Nature (CSRPN). Convaincus de l'intérêt d'étudier un groupe mal connu et souhaitant diversifier leurs approches jusque-là essentiellement tournées vers la gestion de la grande faune, ils ont fait circuler ces documents au sein du Parc. Les agents de terrain ont eux aussi été intéressés. Dès la création du parc national, réaliser des inventaires a été une priorité de l'établissement. Cette priorité avait toutefois été quelque peu délaissée, le retour du loup au début des années 1990 ayant requis une bonne partie de l'énergie du parc national et de son service EGM en particulier. Près de dix ans plus tard, la pression du « dossier loup » a commencé à se desserrer ; l'envie de travailler sur d'autres espèces a augmenté et le Parc a commandé au laboratoire de malacologie du Muséum un inventaire des escargots patrimoniaux présents sur son territoire.

En 2000, les deux structures ont passé une convention d'étude, renouvelée chaque année jusqu'en 2006. Pendant toute cette période, les malacologues du Muséum se sont rendus une semaine par an dans le Parc pour y effectuer des collectes, identifiant ou confirmant les identifications d'escargots opérées par les agents du Parc, et bénéficiant de la connaissance du terrain de ces derniers pour repérer les habitats intéressants pour leurs objets d'étude. Ils ont formé des agents de terrain à la détermination des espèces d'escargots endémiques du 


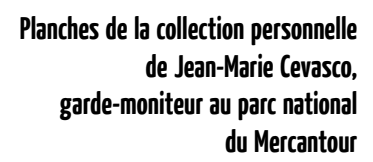

(Fig. 5)

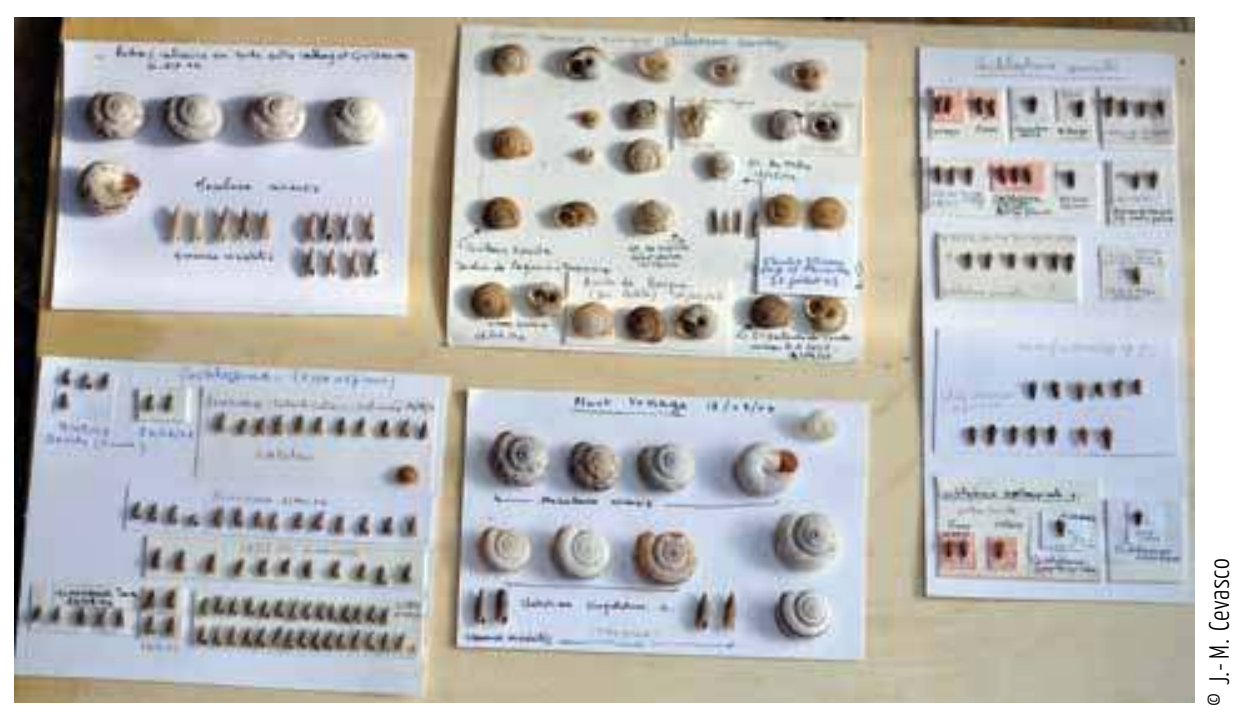

Mercantour et ont élaboré un guide de détermination (Gargominy et Ripken, 2001) (Figure 4). Les agents de terrain ont collecté des coquilles et renseigné des fiches de relevé standardisées. Les coquilles et leurs fiches ont été expédiées au Muséum par le service EGM ou récupérées par les malacologues à l'occasion de leur venue dans le Mercantour. Les agents de terrain ont également constitué des collections de coquilles et de photographies d'escargots dans différents secteurs du Parc, de manière à faciliter le travail de détermination des espèces (Figure 5).

Du point de vue des deux structures, l'inventaire des escargots patrimoniaux « a bien marché. [...] En alliant les qualités de part et d'autre, ça a donné vraiment un bon inventaire. » La coopération entre le Parc et les malacologues du Muséum a en effet permis une nette progression des connaissances : non seulement les espèces et les stations identifiées par les naturalistes un siècle auparavant ont été retrouvées mais d'autres leur ont été ajoutées et des erreurs de nomenclature ont été corrigées. L'expression « bien marché » est de fait très appropriée : elle suggère un mouvement, cette circulation qui nous intéresse ici et, comme cela a déjà été évoqué, l'inventaire a constitué un marché, au sens économique du terme.

L'inventaire a vivement intéressé à la fois des malacologues du Muséum et des agents du Parc. Il a offert aux systématiciens la possibilité, plutôt rare en Europe, de faire progresser leur connaissance de la biodiversité : du fait notamment de sa relative richesse en espèces endémiques, le Mercantour est un terrain intéressant, même s'il ne peut pas rivaliser avec les contrées tropicales où sont organisées les expéditions contemporaines ${ }^{3}$. Côté Parc aussi, l'implication dans l'inventaire a été forte, au siège de l'établissement et sur le terrain. Le chef du service EGM et une de ses collaboratrices se sont investis dans l'organisation et le suivi de l'inventaire. Treize agents de terrain ont participé aux prospections (Figure 6). Certains d'entre eux se sont très fortement mobilisés et ont travaillé « comme des forcenés », selon l'expression d'un enquêté. Eux-mêmes ont été surpris par leur intérêt pour des espèces dont ils avaient jusque-là ignoré l'existence. Apprendre à identifier ces espèces a indéniablement nécessité de leur part un apprentissage et un investissement importants : « il faut quand même vraiment s'y pencher », a sobrement dit l'un d'eux. Les espèces considérées sont en effet de petite taille, au point qu'il faut parfois tamiser l'humus ou le sol pour les isoler et utiliser une loupe binoculaire pour les identifier. Cependant, ces espèces sont relativement plus faciles 
à identifier que d'autres taxons eux aussi méconnus. D'une part, les coquilles se voient tout de même à l'œil nu (Figure 7) ; dans le monde global des escargots, la plupart des escargots patrimoniaux du Mercantour font partie des grandes espèces. D'autre part, tout en étant suffisant pour soutenir l'intérêt, le nombre assez réduit ${ }^{4}$ d'espèces d'escargots endémiques du Mercantour permet d'en devenir spécialiste, moyennant un important investissement. De plus, la détermination des espèces en question est fondée sur l'examen des coquilles (Figure 8). Elle ne nécessite donc pas de prélever ni de détruire des animaux vivants. Les agents de terrain ont beaucoup insisté sur ce point : contrairement à d'autres opérations scientifiques menées dans le cœur du Parc, le travail mené sur les escargots n’a pas heurté leurs engagements professionnels et éthiques en faveur de la protection de la nature. Pour toutes ces raisons (relative richesse en espèces endémiques ; relative facilité d'identification et absence de conflit d'ordre éthique avec les engagements des agents de terrain en faveur de la protection de la nature), l'inventaire des escargots patrimoniaux du Mercantour s'est révélé une entreprise scientifique particulièrement « faisable ( (do-able, Fujimura 1987).

Au terme de ce travail, le Parc et le Muséum ont pu mettre en avant leur capacité à travailler ensemble (Figure 9) et l'utilité de ce type d'inventaire et de la systématique en général pour le Parc. Cette expérience a constitué un atout précieux lorsque le parc national du Mercantour et le parc naturel voisin des Alpi marittime ont présenté au réseau d'excellence EDIT (European Distributed Institute of Taxonomy) leur candidature pour accueillir le premier All Taxa Biodiversity Inventory (ATBI) européen, avec le soutien et l'appui des malacologues du Muséum. L'inventaire des escargots n’a pas seulement précédé l'ATBI ; il l'a véritablement préparé, ouvrant une voie d'entrée efficace dans l'exploration généralisée de la biodiversité toujours en cours dans les deux parcs du Mercantour et des Alpi marittime.

Bien que la coopération entre les deux structures ait ainsi été étroite et couronnée de succès, les pratiques et les visées des systématiciens et des agents du Parc sont demeurées nettement distinctes. Tous ont cherché à la fois à connaître et à protéger les escargots endémiques mais ces deux objectifs nont pas revêtu une égale importance pour les uns et pour les autres. Les systématiciens ont voulu avant tout produire des connaissances académiques tandis que la priorité des agents du Parc a été de protéger les espèces et leurs habitats. Un agent très investi dans l'inventaire a spontanément raconté son intervention in extremis pour empêcher la destruction d'une paroi rocheuse, en bord de route, où il venait de retrouver la présence d'une population d'escargots endémiques découverte cent ans auparavant. En revanche, il est arrivé aux malacologues du Muséum de collecter des escargots vivants, de manière à pouvoir pratiquer des analyses génétiques. L’ horreur » qu’un tel

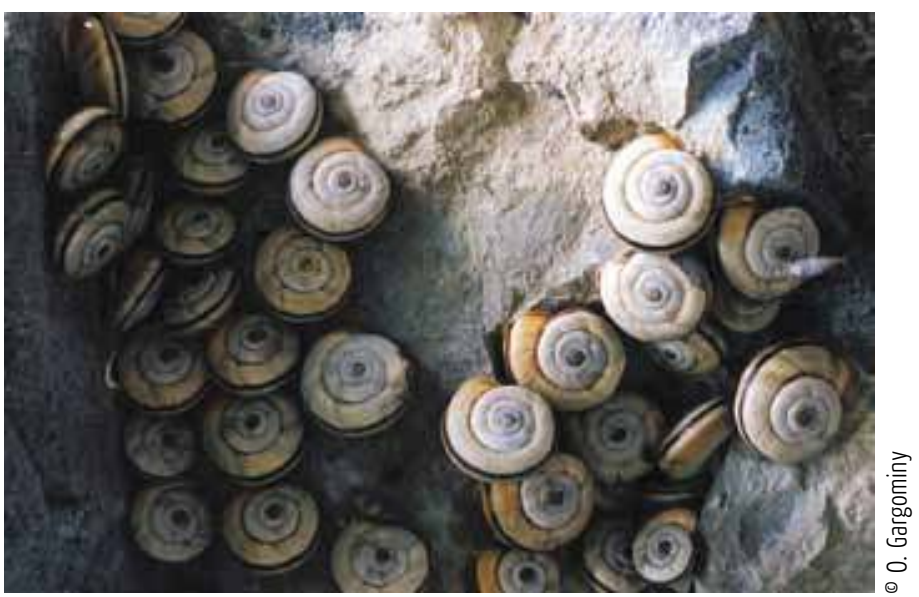




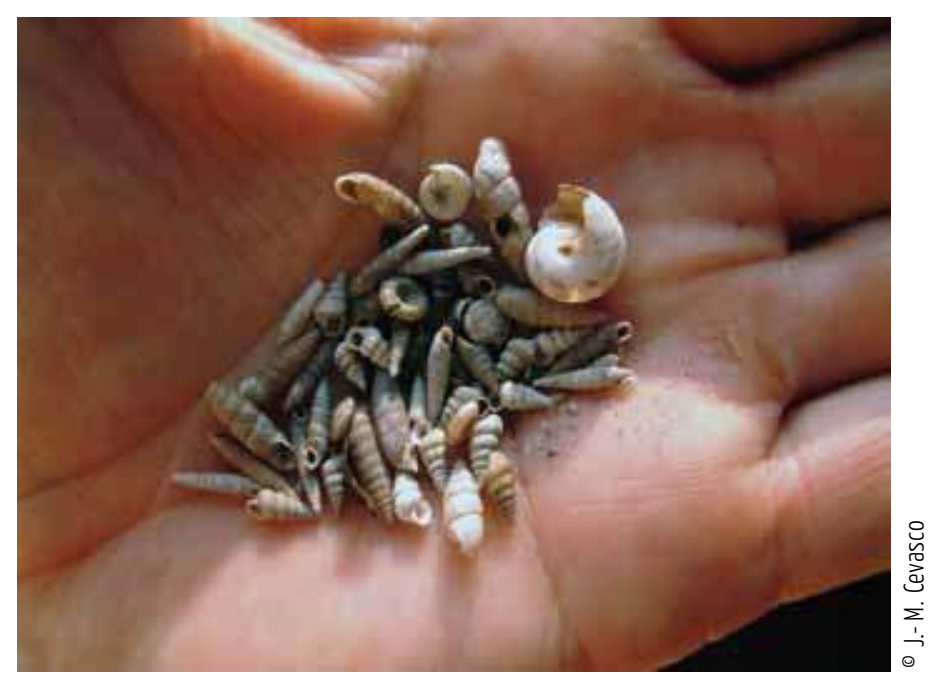

Collecte de coquilles par un agent du Parc

(Fig. 8) geste a inspirée à un garde-moniteur du Parc témoigne bien de la différence d'objectifs et de pratiques entre les deux mondes : « Ils ramassent des animaux vivants. Ils peuvent en ramasser des centaines. Même lorsque l'on est sur une station très rare d'espèces endémiques et très localisée sur quelques mètres carrés, je les ai vus devant mes yeux horrifiés tout ramasser. Mais vraiment tout. C'est-à-dire que tant qu'il y en avait un, ils le prenaient. ${ }^{5}$ »

L'analyse de Star et Griesemer permet de bien comprendre comment une coopération réussie a pu s'établir en dépit de ces différences : grâce à l'élaboration de méthodes standardisées de relevé et d'étiquetage des escargots et grâce à plusieurs types d'objetsfrontières, dont les coquilles d'escargots et les fiches de relevé. Mais il faut aussi saisir l'organisation pratique de la mobilisation de ces objets-frontières. Éclairer cette organisation et pointer ses différentes dimensions constitue l'objet de la partie suivante.
Une séquence de formation des agents du Parc à l'identification des escargots patrimoniaux, par le malacologue Theo Ripken (à gauche, le stylo à la main)

(Fig. 9)

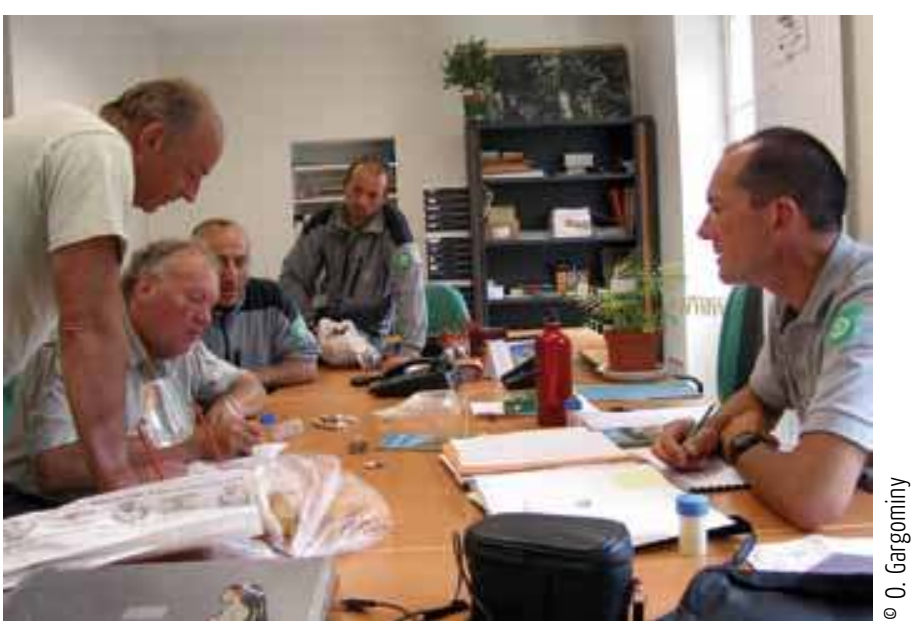

Il ne suffit pas que des systématiciens et des agents du Parc s'intéressent aux escargots endémiques du Mercantour pour les mettre en mouvement et en faire des objets scientifiques. Loin de s'opérer spontanément, la mobilisation des escargots entre le Parc, dans le Mercantour, et le Muséum, à Paris, requiert toute une organisation, dans laquelle le service EGM du Parc a joué un rôle majeur. Cette organisation revêt plusieurs dimensions - matérielle, institutionnelle et marchande - que nous examinerons successivement. La circulation virtuelle des escargots, sur Internet, semble à première vue s'effectuer plus librement. Les voies qu'empruntent les descriptions et les photographies sur les supports numériques ne sont pourtant pas moins étroites ni balisées que celles suivies par les coquilles et les fiches de relevé.

\section{Les dimensions matérielle, institutionnelle et marchande de l'organisation}

Du point de vue matériel, la mise en circulation des escargots est relativement plus simple que celle d'autres taxons. La détermination étant fondée sur l'examen des coquilles, elle ne nécessite pas de conserver des tissus 
vivants $^{6}$ : des coquilles vides sont expédiées, que le déplacement n'altère pas ${ }^{7}$. Des précautions doivent malgré tout être prises pour éviter que les coquilles ne soient endommagées : leur taille réduite permet de les enfermer dans des tubes en plastique rigide, avec un bout de papier ou de coton pour éviter qu'elles ne s'entrechoquent ou dans des petits sachets si les coquilles sont un peu plus volumineuses. Des indications codifiées sur le lieu, la date et l'auteur de la collecte sont étiquetées sur le contenant. Les agents de terrain ont envoyé ou déposé les produits de leur collecte au service EGM du Parc, situé au siège de l'établissement, à Nice. C'est le service qui s'est chargé de grouper les envois au Muséum et d'expédier les colis. Les coquilles devenues objets de science n'ont pas voyagé seules. Elles ont été systématiquement accompagnées d'une fiche (dont le modèle utilisé par les agents du Parc a été élaboré conjointement par le Parc, avec la collaboration d'un agent du service EGM et d'un agent de terrain, et par le Muséum), habituellement remplie au moment de la collecte, qui porte un ensemble d'informations standardisées sur la station et la collecte (Figure 10). C'est la circulation simultanée des coquilles et des fiches de relevé qui en fait ce que Latour (1989) a appelé des « mobiles immuables », garantissant la possibilité, du moins en principe, de revenir du Muséum - « centre de calcul » où les coquilles et les fiches de relevé s'accumulent - jusqu'au lieu de collecte ${ }^{8}$.

Le service EGM a été un acteur discret mais essentiel de la mobilisation des escargots et des fiches de relevé. C'est lui, en effet, qui a notamment pris en charge les aspects logistiques de cette mobilisation. Il a préparé la venue des systématiciens sur le terrain et la formation des agents de terrain et il s'est chargé de l'expédition groupée des coquilles. Le service a également réceptionné les rapports des systématiciens et a élaboré et suivi les conventions annuelles entre le Parc et le Muséum qui ont, dans une large mesure, régi les échanges d'informations et de coquilles entre les membres des deux institutions. Les échanges entre agents de terrain et systématiciens ont été orchestrés par le service ou sont passés par son intermédiaire, même si des échanges directs ont parfois eu lieu.

Le responsable du service alors en poste souligne que le paiement du laboratoire n'a pu s'effectuer sans l'organisation et la formalisation des échanges :

« On avait une convention financière avec le Muséum et le fait de grouper les envois, de centraliser, nous permettait de justifier du fait qu'on finance, qu'on paye effectivement. Si tout était envoyé directement, on n'avait plus de trace du travail demandé au Muséum. Et ensuite, le Muséum nous faisait des rapports. Il nous envoyait des bases de données. Et c'est sur ces bases-là qu'on pouvait payer. »

De l'argent ${ }^{9}$ a circulé du Parc au Muséum, en même temps que les coquilles et les fiches de relevé. Il y a donc bien, aussi, une dimension marchande de la mise en circulation des escargots. Les termes du marché ont été les suivants : le Parc a procuré au laboratoire de malacologie de l'argent et des coquilles d'espèces endémiques accompagnées de fiches de relevé, collectées et remplies par ses agents ; en échange, les malacologues du laboratoire ont formé les agents de terrain à l'identification des escargots patrimoniaux et ont fourni au Parc des rapports contenant des données scientifiquement validées.

\section{Règles et accidents de circulation}

La transformation des coquilles en objets de science a été rendue possible par leur mobilisation et par l'organisation matérielle, institutionnelle et marchande qui sous-tend cette mobilisation. Les coquilles d'escargots et les fiches de relevé n'ont pas circulé n'importe où ni n'importe comment, mais selon des règles et des itinéraires plus ou moins clairement 


\section{Une fiche remplie par un agent de terrain}

(Fig. 10)

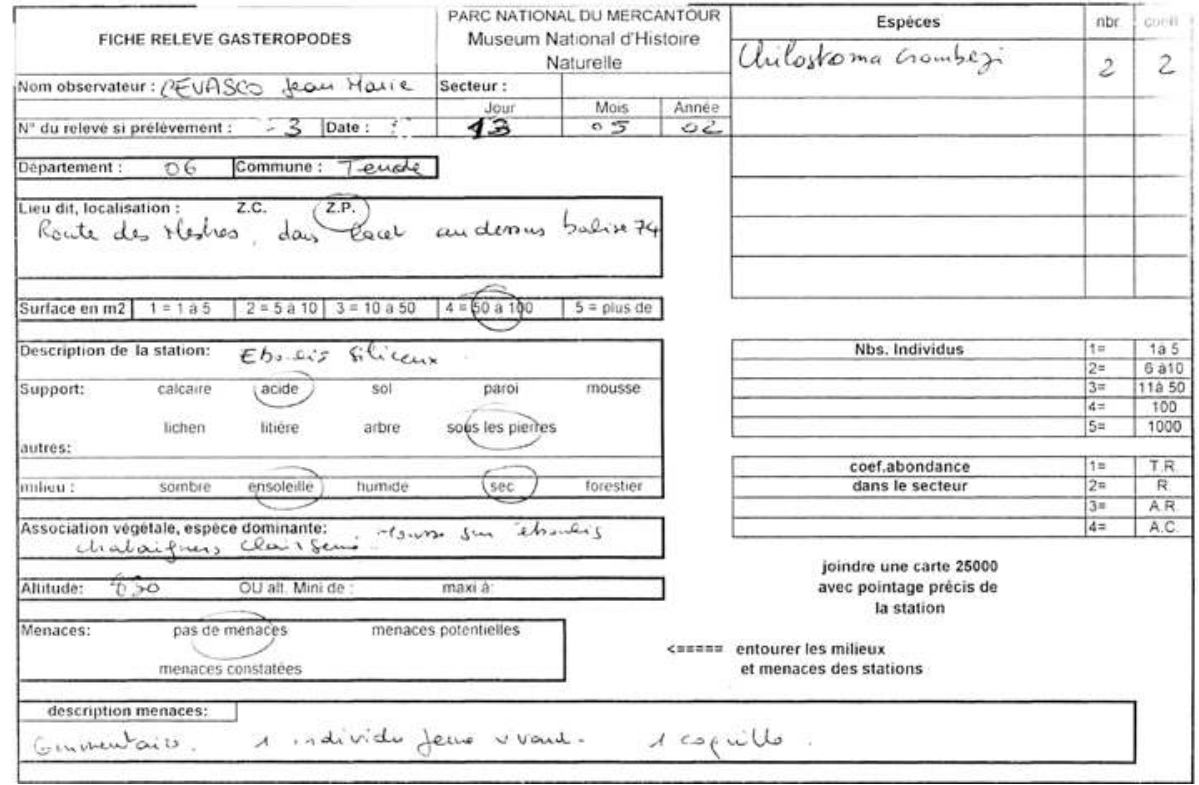

définis. En particulier, les coquilles et les fiches de relevé ont obligatoirement voyagé ensemble et ont constitué, en quelque sorte, un unique objet de circulation et de connaissance. Sauf exception, elles sont par ailleurs passées via le service EGM du Parc, étape indispensable à leur transformation en marchandise scientifique et à la poursuite de l'entreprise.

Bien que ces conditions de circulation n'aient pas été nécessairement explicitées, elles ont constitué des règles dont le non-respect a pu entraîner un arrêt de la circulation des coquilles et des fiches:

« J'ai fait beaucoup, beaucoup de fiches et c'est là où je... enfin, je n'étais pas content parce que j'ai fait peut-être 200 fiches d'espèces patrimoniales avec de nouvelles stations et ils n'ont pas voulu les prendre parce que je n'avais pas joint les coquilles avec... alors que là, j'étais absolument certain de mes déterminations. Les mêmes espèces avaient déjà été validées avec eux in situ ; j’avais mes références de collection validées, ça ne servait à rien que je complique la chose en mettant en plus une éprouvette, chaque fois, dans une pile de 200 feuilles. Et donc j'ai eu deux ans de travail qui sont partis à la trappe. Je l'ai vécu comme un manque total de confiance. »

Les fiches remplies par les agents et non accompagnées de coquilles de même que les coquilles sans fiche ont été écartées du circuit et ne sont pas devenues objets de science. Leur circulation a été brusquement interrompue et elles ont complètement divergé de la trajectoire idéale, censée les conduire du Mercantour au Muséum. Mais des fiches et des coquilles peuvent aussi être égarées ou ignorées. es gardes-moniteurs ont rapporté que certaines de leurs fiches ont été remplacées par des fiches établies par les systématiciens venus observer directement les stations décrites par les agents. Pertes, oublis, retraits de coquilles sans fiche et de fiches sans coquille, substitution d'une fiche par une autre : ces exemples témoignent du fait que la circulation des objets scientifiques peut bifurquer, connaître un coup d'arrêt ou un retour en arrière. Elle peut connaître, en bref, des accidents de parcours.

Les coquilles d'escargots ne sont pas d'emblée des marchandises scientifiques : elles sont transformées en étant transportées d'un lieu et d'un acteur à un autre, le long de 
voies le plus souvent étroitement balisées. La troisième partie du texte porte sur ces transformations, qui concernent à la fois les objets scientifiques et ceux qui les font circuler.

\section{Les effets de la mise en circulation des escargots}

Les coquilles et les fiches de relevé parvenues au terme de leur itinéraire ont acquis une valeur scientifique: les espèces ont été déterminées, nommées, mises en collection et leur statut de conservation a été précisé, en référence aux typologies en vigueur ${ }^{10}$; les données recueillies ont été entrées dans une base de données spécifique, intitulée MALACO-FR ${ }^{11}$. Mais les escargots dont les coquilles et les fiches ont circulé ne sont pas seulement devenus des objets de science. Ils ont aussi acquis une valeur esthétique. Les malacologues du Muséum et des agents du Parc ont de superbes photographies d'escargots (Figures 11 et 12). Or, ces espèces ne sont pas particulièrement belles ni photogéniques à première vue. Habitués à des espèces plus spectaculaires, les agents du Parc ont d'ailleurs dit avoir été déçus, dans un premier temps, par ce qu'il s'agissait d'examiner. La beauté des photographies d'escargots n'est donc pas donnée d'emblée. Elle repose sur un travail et une mise en scène : les malacologues photographes s'appliquent à

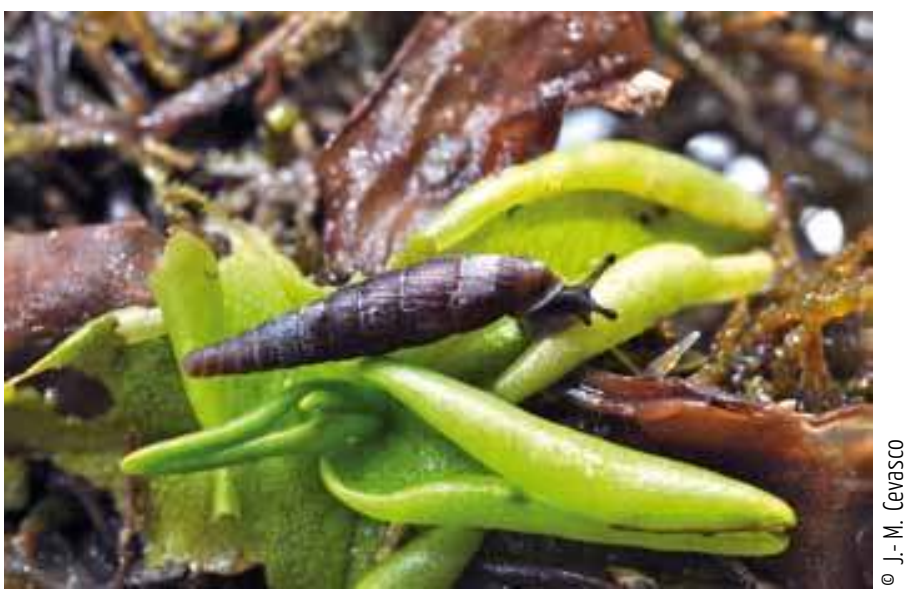

l'esthétisation des escargots mettre en valeur une beauté qui ne saute pas aux yeux. Ainsi, les escargots patrimoniaux du Mercantour sont devenus à la fois des marchandises scientifiques et des objets esthétiques.

Leur mobilisation a été indispensable à ces transformations. Une fois arrivées au Muséum, les coquilles et les fiches, cependant, ne se sont plus déplacées qu'exceptionnellement. Lors de ma visite au laboratoire de malacologie, j’ai été autorisée à voir la collection nationale de référence et à photographier des spécimens du Mercantour sans être accompagnée, mais contre l'assurance expresse que les coquilles extraites de leur tiroir seraient toutes remises en place. Le statut d'objet de collection scientifique acquis par certaines coquilles restreint considérablement la possibilité de les remettre en circulation $^{12}$. Contrairement à celui de marchandise (Kopytoff 1986), ce statut, une fois acquis, est particulièrement difficile à perdre et oriente la biographie de l'objet pour une durée indéterminée : une collection naturaliste est faite pour durer. Les photographies les plus réussies, elles, continuent au contraire de circuler. Permise par le déplacement, la transformation des escargots exerce en retour un effet sur les déplacements futurs, qui dépend de la nature de la transformation effectuée.

Par ailleurs, le retrait des fiches sans coquille mentionné précédemment témoigne du fait que la transformation en objets de science requiert, de fait, l'intervention d'une personne autorisée. Les transformations des escargots sont ainsi liées au statut de ceux qui les font circuler. Mais ces derniers aussi ont changé. Les agents de terrain les plus investis dans l'inventaire ont expliqué

\section{Macrogastra mellae leia Chilostoma crombezi}

(Fig. 11 et 12) 
avoir éprouvé un intérêt renouvelé pour un secteur de montagne qu'ils parcouraient depuis des années, en découvrant, « à leur porte », des espèces et des habitats dont ils ne soupçonnaient pas l'existence. Certains se sont véritablement « passionnés », à leur propre surprise, pour la recherche et la détermination des escargots patrimoniaux (Figure 13). Outre qu'elle leur a permis de porter un regard neuf sur leur terrain, leur participation à l'inventaire a fait d'eux des découvreurs de nouvelles stations et des spécialistes de ces espèces. Ces personnes ont largement souligné le fait qu'elles n'ont pas uniquement servi de guides aux systématiciens et que leur rôle ne s'est pas cantonné à collecter des coquilles et à approvisionner en objets scientifiques le « centre de calcul » (Latour 1989) qu'est le Muséum. Redécouvrir un terrain qu'ils croyaient connaître parfaitement et devenir des spécialistes d'espèces méconnues et des découvreurs de nouvelles stations: ces changements ont constitué des motivations importantes pour s'impliquer dans l'inventaire.

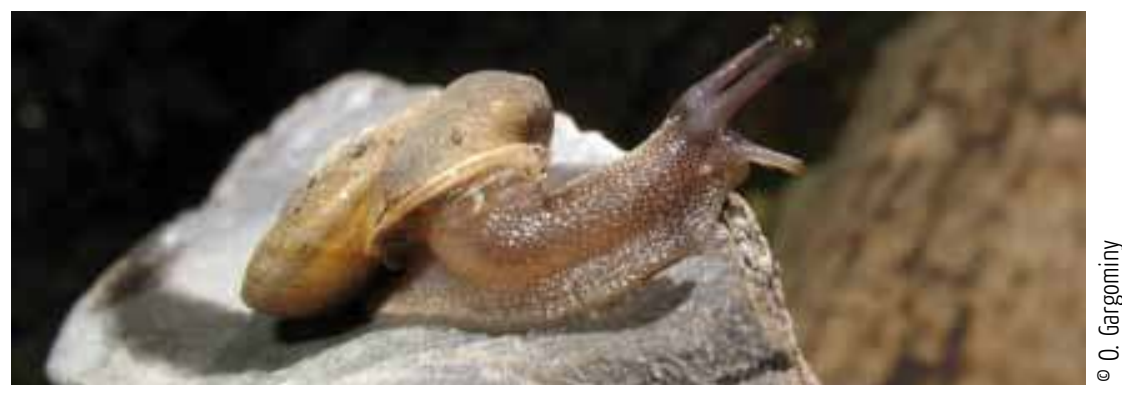

Dans ces conditions, les accidents de parcours mentionnés précédemment n'ont pas été sans conséquences pour les personnes qui ont fait circuler les coquilles et les fiches de relevé et pour les interactions entre ces personnes. L'inventaire, à nouveau, a été une réussite pour les deux structures et a largement favorisé le lancement de l'ATBI MercantourAlpi marittime. Des frictions ont cependant eu lieu. Elles ont été suffisantes, dans un cas au moins, pour conduire à des accusations de « récupération » du travail des agents de terrain et à l'arrêt semble-t-il définitif de la collaboration. Pourtant, dans l'article qui a suivi l'inventaire, les malacologues du Muséum ont explicitement remercié les agents du Parc, collectivement dans le corps du texte et nominativement dans la rubrique « Remerciements». Les agents apparaissent aussi dans la liste des collectes effectuées. Ils n'ont certes pas été signataires de l'article, ce qui indique que leur contribution n'a pas justifié, aux yeux des systématiciens, qu'ils apparaissent comme des auteurs du texte ${ }^{13}$ (Pontille 2007) mais ils n'ont pas été non plus dans la situation des «techniciens invisibles » de la science (Shapin 1989) : leur contribution n’a pas été effacée de la présentation publique de l'inventaire et son importance a été reconnue ${ }^{14}$. Mais le retrait des fiches sans coquilles ou la substitution d'une fiche de relevé par celle d'un autre observateur ont été vécus comme un blocage, non seulement des objets concernés, mais de ceux qui les avaient mis en circulation. Tout s'est passé comme si le retrait et la substitution des fiches avaient été assimilés à un rejet de l'accession des gardes-moniteurs au statut d'identificateurs fiables d'espèces et de découvreurs de nouvelles stations et à un maintien dans un statut de guide de terrain et de collecteur. Les frictions entre les personnes ont 
ici tenu aux frottements et aux flottements dans la mobilisation des objets, ce qui suggère que l'invisibilisation des présences (Bidet et Schoeni, 2011) est moins en cause que celle des déplacements.

\section{$\&$}

Loin d'être totalement improvisée et contingente, la mise en circulation des objets scientifiques est organisée et suit un ensemble de règles. En l'occurrence, les coquilles d'escargots patrimoniaux collectées sur le terrain devaient être envoyées au siège du Parc, accompagnées d'une fiche de relevé standardisée, puis expédiées dans des colis groupés au Muséum national d'histoire naturelle.

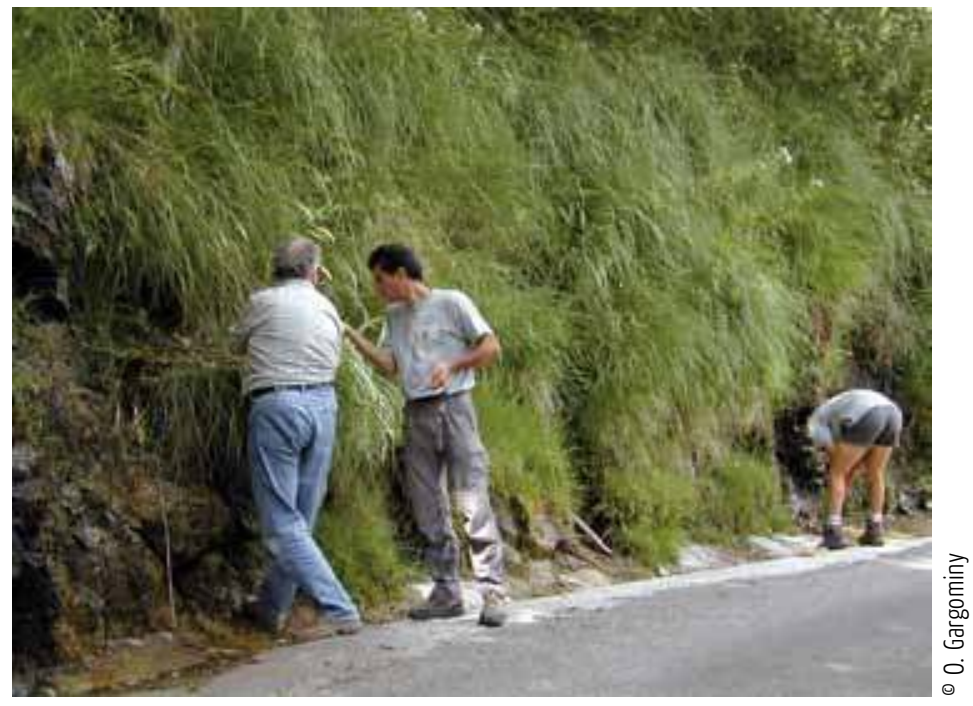

Là, des coquilles devaient être mises en collection et les données en base de données.

Séquence de prospection

(Fig. 13) Mais cette trajectoire idéale a connu des inflexions et des interruptions. Des coquilles sont restées dans le Mercantour pour constituer des collections locales et faciliter l'apprentissage des critères de détermination par les agents du Parc : la coopération entre des mondes sociaux différents, qui rend possible le cycle d'accumulation des connaissances, suppose que tous les objets ne rejoignent pas les centres de calcul et soient aussi étudiés sur place, par des personnes qui en deviennent des spécialistes locaux. L'enquête a aussi mis en évidence l'existence de quelques accidents, qui ont interrompu le parcours de fiches et de coquilles, à différents stades et pour différentes raisons. Ces accidents de parcours ont bloqué les transformations qui s'opèrent dans le cours de la mobilisation des objets scientifiques : les coquilles et les fiches en question ne sont pas devenues des objets de science ; les agents qui les avaient collectées et remplies ne sont pas devenus des identificateurs d'espèces et des découvreurs de stations autorisés. Les frictions observées témoignent de l'intérêt de, et de la difficulté à, discuter et à négocier collectivement les modalités de circulation des objets scientifiques, dans un cas où ces frictions sont demeurées limitées et n'ont pas empêché l'établissement d'une coopération considérée comme largement réussie.

Parce que la mise en circulation des objets scientifiques opère des transformations multiples, la coopération qui la sous-tend est nécessairement dynamique. Entre le début et la fin de l'inventaire, les escargots et ceux qui les ont mobilisés ont un peu changé. Les agents de terrain du Parc comme les malacologues du Muséum ont renouvelé leurs connaissances, leurs compétences et leur rapport au terrain. La reconnaissance de ces changements constitue un enjeu majeur pour les personnes impliquées. Toutes aspirent à circuler et à évoluer en même temps que les escargots qu'ils ont contribué à mobiliser. Cette enquête sur l'inventaire des escargots patrimoniaux du Mercantour montre, en définitive, que la mise en circulation des objets scientifiques entre des mondes sociaux différents permet une reconfiguration de ces mondes et de leurs interrelations, au-delà de leur coopération. 


\section{NOTES}

Photo d'ouverture : L'esthétisation des escargots. Chilostoma crombezi. (Cl. Olivier Gargominy)

1. Au sens de rendre mobile. J'emploierai indifféremment mobilisation et mise en circulation.

2. Pour une discussion récente sur la notion d'objet-frontière, voir le numéro spécial de la Revue d'Anthropologie des Connaissances publié en 2010 (vol 4, nº).

3. Sur l'expédition organisée à Santo en 2006, voir Bouchet et al. (2008) ; pour une analyse anthropologique, voir Faugère (2008).

4. Une vingtaine d'espèces de mollusques terrestres patrimoniaux ont été recensées dans la région PACA. Le guide d'identification élaboré par les malacologues du Muséum comporte 13 taxons, sélectionnés pour leur facilité de récolte (coquilles repérables à l'œil nu) et de détermination (détermination fondée sur l'examen des coquilles et l'existence de critères de détermination clairs).

5. Les malacologues du Muséum ont démenti cette affirmation. Ils ont indiqué tenir compte du rapport, variable selon les espèces, entre le nombre d'individus visibles et le nombre total d'individus et limiter leur prélèvement à quelques dizaines d'escargots par site au maximum, de manière à ce que leurs collectes de spécimens vivants ne mettent aucune population en péril.

6. Nombre d'escargots vivants ont toutefois circulé par la poste, plongés en léthargie dans du sopalin bien sec (O. Gargominy, communication personnelle).

7. Ni le temps. Lors du séminaire de préparation de ce numéro (Châteauneuf-de-Gadagne, 25-26/01/12), Philippe Bouchet notait que les fossiles et les coquilles sont les seuls éléments des collections mal conservées à être épargnés par les parasites et les prédateurs.

8. Latour définit les mobiles immuables comme « ces objets [qui] se situent au début et à la fin d'un cycle d'accumulation similaire; peu importe s'ils sont proches ou éloignés, infiniment grands ou infiniment petits, infiniment vieux ou jeunes, ils finissent tous par exister à une échelle que quelques-uns peuvent dominer du regard; à un moment ou à un autre, ils prendront tous la forme d'une feuille de papier qui peut être archivée, épinglée à un mur et combinée à d'autres; ils contribuent tous à renverser l'équilibre des forces entre ceux qui dominent et ceux qui sont dominés » (Latour 1989 : 545). Sur l'importance des mobiles immuables dans les itinéraires de coquillages, voir Dumoulin dans ce numéro.

9. Les moyens financiers engagés sont restés modestes. À titre d'exemple, la convention 2000-2001 prévoyait un versement au laboratoire d'un montant de 15000 francs. Il convient d'ajouter à ces sommes le temps de travail des agents du Parc.

10. En l'occurrence, les systématiciens du Muséum se sont référés à la typologie utilisée pour l'inventaire des Zones naturelles d'intérêt écologique, faunistique et floristique (Znieff).

11. Le Parc a désormais accès aux données, en temps réel, via le site internet de l'Inventaire national du patrimoine naturel (INPN).

12. Sur les règles de prêt des spécimens mis en collection, voir Faugère dans ce numéro.

13. Sur les enjeux de la signature des articles scientifiques, voir Pontille (2007).

1. Les participants à l'inventaire apparaissent également sur la page principale des espèces sur le site internet de l'INPN. Voir, par exemple, http://inpn.mnhn. fr/espece/cd_nom/162979 ou http://inpn.mnhn.fr/ espece/cd_nom/416742 consultés le 9 mars 2013. 


\section{RÉFÉRENCES}

Appadurai, A. (dir.) 1986 The Social Life of Things. Commodities in Cultural Perspective. Cambridge: Cambridge University Press, 329 p.

Bidet, A., Schoeni, D. 2011 Décrire les présences au travail, analyser la structuration de la vie sociale. In ethnographiques.org 23 (décembre 2011) : Analyser les présences au travail : visibilités et invisibilités [en ligne]. (http://www.ethnographiques.org/2011/Bidet,Schoeni - consulté le 21.02.2012)

Bouchet, P., Le Guyader, H., Pascal, O. 2008 Des voyages de Cook à l'expédition Santo 2006 : un renouveau des explorations naturalistes des îles du Pacifique. In Le Journal de la Société des Océanistes 126-127 : 167-186. Mis en ligne le 15 décembre 2011, consulté le 22 janvier 2013. http://jso.revues.org/4622.

Bouchet, P. 1990 La Malacofaune française : endémisme, patrimoine naturel et protection. Revue d'écologie (Terre et Vie) 45 (3) : 259-288.

Faugère, E. 2008 L'exploration contemporaine de la biodiversité. Approche anthropologique de l'expédition Santo 2006. Le Journal de la Société des Océanistes 126-127 : 195-206. Mis en ligne le 15 décembre 2011, consulté le 22 janvier 2013. http://jso.revues.org/4622.

Fujimura, J. H. 1987 Constructing 'Do-Able’ Problems in Cancer Research : Articulating Alignment. Social Studies of Science 17(2) : 257-293.

Gargominy, O., Ripken, T., E.J. 1999 Inventaire des mollusques d'intérêt patrimonial de la région PACA. Programme d'actualisation des ZNIEFF PACA. Aix-en-Provence / Paris : Conservatoire Études des Écosystèmes de Provence / Alpes du Sud - Muséum national d'histoire naturelle.

Gargominy, O., Ripken, T., E.J. 2001 Mollusques continentaux à valeur patrimoniale du Parc national du Mercantour. Nice / Paris : Parc national du Mercantour, Muséum national d'histoire naturelle, Association pour la Connaissance et la Protection du Patrimoine naturel.

Kopytoff, I. 1986 The Cultural Biography of Things : Commoditization as Process. In A. Appadurai (dir.), The Social Life of Things. Commodities in Cultural Perspective. New York : Cambridge University PressKopitoff.

Latour, B. 1989 La Science en action. Paris : La Découverte.

Marcus, G.E. 1995 Ethnography in/of the World System : The Emergence of Multi-Sited Ethnography. Annual Review of Anthropology 24 : 95-117.

O'Connell, J. 1993 The Creation of Universality by the Circulation of Particulars. Social Studies of Science 23(1) : 129-173.

Pontille, D. 2007 Générique de noms et évaluation du travail scientifique. In F. Gaudez Sociologie des arts, sociologie des sciences (tome 1). Paris : L'Harmattan : 225-241.

Shapin, S. 1989 The Invisible Technician. American Scientist 77 : 554-563.

Star, S. L., Griesemer, J. R. 1989 Institutional Ecology, "Translations" and Boundary Objects: Amateurs and Professionals in Berkeley's Museum of Vertebrate Zoology. Social Studies of Science 19(3) : 387-420.

\section{REMERCIEMENTS}

Cette recherche a été menée dans le cadre du projet PAN-bioptique, financé par le programme « Sciences, technologies et savoirs en société » de l'ANR. Je remercie l'ensemble des personnes enquêtées ainsi qu'Olivier Gargominy, Benoît Lequette, Marie-France Leccia, Monique Perfus, Jean-Marie Cevasco et Raphaël Larrère pour leurs commentaires sur une version antérieure de ce texte. Je reste bien sûr seule responsable de l'interprétation et des erreurs éventuelles. 


\section{RÉSUMÉ}

La mise en circulation des objets scientifiques : organisation pratique et changements de statut. Le cas des escargots patrimoniaux du Mercantour. Cet article réfléchit à l'organisation et aux effets de la mise en circulation des objets scientifiques entre des mondes sociaux différents à partir de l'exemple des escargots patrimoniaux du Mercantour. Il s'appuie sur une enquête de terrain, qui a visé à retracer les itinéraires des coquilles d'escargots, du Mercantour au Muséum national d'histoire naturelle et à saisir les transformations qui les ont accompagnés. Je commence par introduire les deux notions sociologiques principalement utilisées : celle d'objet-frontière et celle de vie sociale des choses, que j'emprunte respectivement aux travaux classiques de Star et Griesemer (1989) et d'Appadurai (1986). Je présente ensuite la genèse et le déroulement de l'inventaire des escargots patrimoniaux du Mercantour, dans lequel des malacologues du Muséum et des agents du parc national du Mercantour ont étroitement collaboré. Je m'intéresse alors à l'organisation pratique de la mise en circulation des coquilles et des fiches de relevé qui les accompagnent. La transformation des coquilles en objets de science a été rendue possible par leur mobilisation et par l'organisation matérielle, institutionnelle et marchande qui sous-tend cette mobilisation. Des règles plus ou moins clairement définies ont régi la circulation des coquilles et des fiches de relevé, qui a aussi été marquée par quelques accidents de parcours : les coquilles et les fiches n'ont pas toujours suivi la trajectoire idéale. La dernière partie porte sur les changements de statut opérés par la mobilisation des objets scientifiques. Ces changements de statut concernent à la fois les objets scientifiques et ceux qui les mettent en mouvement. Ils constituent une motivation forte de l'implication dans l'entreprise scientifique et l'invisibilisation des déplacements effectués peut alors constituer une importante source de friction entre les contributeurs à cette entreprise. L'article montre en définitive que la mise en circulation d'objets-frontières ne permet pas seulement la coopération entre des mondes sociaux différents mais contribue également à la reconfiguration de ces mondes et de leurs interrelations.

\section{ABSTRACT}

The circulation of scientific objects: practical organization and changes in status. The case of the Mercantour's rare snails. This article draws on the case of the Mercantour's rare snails to reflect on the modalities and effects of the circulation of scientific objects between different social worlds. It is based on a field survey that aimed to retrace the routes of the snail shells, from the Mercantour to the National Museum of Natural History, and to grasp the transformations that took place along these routes. I begin by presenting the two main sociological notions I use: that of "boundary object" (Star and Griesemer 1989) and that of "social life of things" (Appadurai 1986). I then present the genesis and development of the inventory of rare snails in the Mercantour, in which malacologists from the Museum and staff from the Mercantour national park cooperated very closely with one another. Next, I turn to the practical organization of the circulation of the shells and forms that accompany them. This circulation and the material, institutional and market organization that underpinned it allowed the transformation of the shells into scientific objects. While more or less clearly defined rules governed the circulation of shells and forms, some accidents occurred along the way: the shells and forms did not always follow the ideal trajectory. The last part of the article deals with the changes in status performed by the circulation of scientific objects. These changes concern both the scientific objects and those who circulate them. They are a strong motivation to become involved in the scientific undertaking and rendering movements invisible can thus generate an important amount of friction between the participants. Overall, the article shows that the circulation of scientific objects does not only allow the cooperation between different social worlds but also contributes to the reconfiguration of these worlds and their interactions. 


\section{MOTS CLÉS}

Escargots, inventaire naturaliste, objet scientifique, objet-frontière, vie sociale des choses, mise en circulation, Mercantour, Parc national, Muséum national d'histoire naturelle.

\section{KEYWORDS}

Snails, naturalist inventory, scientific object, boundary-object, social life of things, circulation, Mercantour, national Park, National Museum of Natural History. 